\title{
LA METODOLOGÍA LÚDICA COMO DINAMIZADOR DE LAS CONDUCTAS PROSOCIALES
}

LUDIC METHODOLOGY AS A PROMOTER OF PROSOCIAL BEHAVIOR

\section{A METODOLOGIA RECREATIVA COMO DINÂMICA DE COMPORTAMENTOS PRÓ-SOCIAIS}

\author{
Greys Núñez-Ríos ${ }^{1}$ \\ Edgardo Márquez-Núñez ${ }^{2}$ \\ Hilda Guerrero-Cuentas ${ }^{3}$ \\ Marta Magdaniel-Durán ${ }^{4}$ \\ Bielenys Silvera-Goenaga ${ }^{5}$
}

\section{Resumen}

El presente artículo de investigación tiene como objetivo presentar los avances del fortalecimiento de las conductas prosociales a través de la metodología lúdica. Se abordan referentes teóricos como Moñivas (1996), Auné y otros (2014) con relación a conductas prosociales, y Espinoza y Rodríguez (2017) en referencia a la lúdica como estrategia dentro del proceso de enseñanza-aprendizaje en la educación básica primaria. La metodología utilizada se enmarca en el paradigma sociocrítico, con un enfoque introspectivo vivencial, de tipo cualitativo y un diseño de investigación-acción de alcance descriptivo, con el que se pretende reconstruir la realidad partiendo del entorno social, observando los fenómenos que ocurren de manera flexible para entender, interpretar y analizar los resultados, avances y dificultades presentados por los estudiantes con relación a la caracterización inicial y final. De la ejecución de las estrategias se concluye que estas generan en los estudiantes cambios conductuales que se ven reflejados tanto en el aspecto vivencial como académico fortaleciendo su formación integral que le permitirá desempeñarse como ciudadanos de mundo.

Palabras clave: comportamiento humano; juego educativo; método de enseñanza

\section{Abstract}

The purpose of this research article is to present the advances in the strengthening of prosocial behaviors through the ludic methodology. They were taken theotherical referent as Moñivas, Auné and others in relationship with prosocial behaviors and Espinoza and Rodriguez (2017) in reference to the ludic as strategy in the teaching learning process in primary basic education. The methodology used is part of the sociocritic paradigm, with an experiential introspective approach of qualitative type and a design of action research with a descriptive scope with which it is intended to rebuild the reality from the social environment, observing the phenomena that occur flexibly to understand, interpret and analyze the results, advances and difficulties pre-

1 Magister en Educación, Universidad Autónoma del Caribe. Docente medio tiempo, Universidad de la Costa, Barranquilla, Colombia. Correo: gnunez4@cuc.edu.co. Orcid: 0000-0001-6426-27292

2 Magíster en Educación, Universidad de la Costa. Docente medio tiempo, Universidad de la Costa, Barranquilla, Colombia. Correo: emarquez12@cuc.edu.co. Orcid: 0000-0001-6505-93003

3 Doctora en Educación, Universidad Rafael Belloso Chacín, Maracaibo, Venezuela. Docente Medio Tiempo, Universidad de la Costa, Barranquilla, Colombia. Correo: hguerrero@cuc.edu.co. Orcid: 0000-0002-8135-75194

4 Licenciada en Básica Primaria, Universidad de la Costa, Barranquilla, Colombia. Correo: marisbel01@gmail.com. Orcid: 0000-0002-6296-52925

5 Licenciada en Básica Primaria, Universidad de la Costa, Barranquilla, Colombia. Correo: bielenisk@gmail.com. Orcid: 0000-0002-0372-7112 
sented by the students in relation to the initial and final characterization. Based on the implementation of the strategies, it may be concluded that these ones generate in the students behavioral changes that are reflected in both the experiential and academic aspects, strengthening their integral education that will allow them to function as world citizens.

Key words: human behavior; educational games; teaching method

\section{Resumo}

Este artigo de pesquisa tem como objetivo apresentar o progresso do fortalecimento de comportamentos pró-sociais por meio de metodologia lúdica. São abordadas referências teóricas como Moñivas (1996), Auné e outros em relação aos comportamentos pró-sociais e Espinoza e Rodríguez (2017) em referência à lúdica como estratégia no processo de ensino-aprendizagem no ensino fundamental. A metodologia utilizada é enquadrada dentro de um paradigma sócio-crítico, com abordagem experiencial introspectiva, de tipo qualitativo e delineamento de pesquisa-ação de escopo descritivo, com o qual se pretende reconstruir a realidade com base no ambiente social, observando os fenômenos que ocorrem de maneira flexível para entender, interpretar e analisar os resultados, avanços e dificuldades apresentados pelos alunos em relação à caracterização inicial e final. A partir da execução das estratégias, conclui-se que elas geram mudanças comportamentais nos alunos que se refletem nos aspectos experienciais e acadêmicos, fortalecendo sua formação integral que lhes permitirá atuar como cidadãos do mundo.

Palavras-chave: comportamento humano; jogo educativo; método de ensino

Para citar este artículo:

Núñez Ríos, G., Márquez Núñez, E., Guerrero Cuentas, H., Magdaniel Durán, M., y Silvera Goenaga, B. (2019). La metodología lúdica como dinamizador de las conductas prosociales. Lúdica Pedagógica, 30, 21-29.

https://doi.org/10.17227/ludica.num30-11101 


\section{INTRODUCCIÓN}

La conducta prosocial en el ámbito educativo se constituye en los momentos actuales en un componente esencial para toda la comunidad integrante de una escuela, en los diversos niveles o grados de estudio. Facilita las interacciones efectivas entre los estudiantes y fomenta principios y valores éticos, que posibilitan la ayuda, la colaboración y el apoyo mutuo. Desde el origen epistemológico la conducta prosocial, según Moñivas (1996):

Es una de las formas del comportamiento moral, las cuales se han asociado, en las distintas investigaciones, con las conductas de consuelo, dar, ayuda, altruismo, confianza, compartir, asistencia, cooperación siendo la última en venir a escena la conducta de solidaridad (p. 127).

Esta variedad de acciones hace que la definición puntual sea compleja de precisar. Sin embargo, el término de conducta prosocial fue incluido, históricamente en las ciencias sociales durante la época de los años setenta, para hacer referencia a una conducta positiva social, pero sin hacer mención necesariamente a una intención altruista. Por eso, toda conducta altruista es una conducta prosocial, pero no toda conducta prosocial es altruista.

De acuerdo con lo anterior, las conductas prosociales son voluntarias, forman parte del ser humano y no son impuestas por la sociedad. Así mismo, estas conductas se generan en el yo, a partir de la necesidad que se ve en la otra persona. Es decir, la conducta intrínseca motiva a una buena relación con el otro lo que fortalece la afectividad y la convivencia dentro de un colectivo (Auné, Blum, Abal, Lozzia, Horacio, 2014).

Por lo tanto, se considera conducta prosocial toda conducta positiva con o sin motivación altruista, entendiendo por positiva que no daña, que no es agresiva. A su vez, se asume que la motivación altruista es el deseo de favorecer al otro con independencia del propio beneficio. Es decir, que la prosocialidad gira en torno a la ayuda sin esperar nada a cambio, lo cual deriva en el crecimiento personal del individuo que asuma esta conducta, de ahí la importancia de empezar a aplicar este tipo de proyectos en todas las instituciones educativas del país, ya que se aportaría al fortalecimiento de las relaciones interpersonales sanas en la sociedad.

En el ámbito educativo, el fortalecimiento de las conductas prosociales es una temática interesante para la comunidad académica nacional e internacional, por todo lo que implica establecer relaciones afectivas en el aula, como parte de la formación integral. Además, la manera como se implementa la lúdica aporta elementos importantes en las estrategias didácticas, que son un factor esencial en la acción educativa. Según Espinoza y Rodríguez (2017):

Se requiere, tarea fundamental del profesor como mediador o facilitador, generar un clima social que propicie la sana y asertiva relación y el establecimiento de vínculos directivos/alumnos, profesor/ alumno, alumno/alumno, pues en esta habilidad social residen las condiciones del aprendizaje autónomo y colaborativo para lograr la potencialización de las habilidades de los jóvenes y con ello garantizar el desarrollo de competencias (p. 3).

En este sentido, la manera como se implementa la lúdica aporta elementos importantes en las estrategias didácticas, de forma tal que los ambientes de aprendizaje favorezcan el robustecimiento de la convivencia en el aula, desde la educación básica primaria, porque es el momento en que se descubren y desarrollan las habilidades emocionales necesarias para la identificación y respuesta constructiva de las emociones propias y las de los demás.

De acuerdo con lo anterior, potencializar estas conductas favorecen los espacios de convivencia y la formación en valores como la tolerancia, el respeto al prójimo, solidaridad, cooperación, el trabajo en equipo, el reconocimiento de las habilidades y competencias individuales para alcanzar objetivos grupales, el desarrollo de la autoeficiencia, la promoción del desarrollo, las interacciones sociales entre pares $y$, sobre todo, el descubrimiento de las destrezas de cada individuo que le permite conocer lo que es capaz de hacer para lograr una meta en común.

En las instituciones educativas, es común observar la formación de grupos sociales dentro y fuera del aula de clases, donde los estudiantes se unen con quienes tienen más afinidad, esto hace parte de la interacción con otros individuos, en muchas ocasiones estas relaciones pueden ser positivas o negativas. Cuando son positivas "van aprendiendo cómo comportarse 
en determinadas situaciones, cómo jugar en grupo, cómo hacer amigos y también aprenden acerca de otras personas" (Casado, 1984, p. 63).

Cuando las relaciones son negativas, se forman juicios u opiniones no favorables de los individuos por el hecho de no tener afinidad con los demás, allí entra el rechazo, la discriminación, entre otros aspectos, los cuales traen como consecuencia malas relaciones entre compañeros, afectando el ambiente escolar, la calidad educativa, la promoción del aprendizaje, la sana convivencia y la cultura de paz.

De acuerdo con la intencionalidad establecida para dinamizar las conductas prosociales en el aula, Domínguez afirma:

Resulta imprescindible que la educación actual, se apoye en la evolución psicológica del juego infantil, siguiendo las fases de su desarrollo, incluyendo al aspecto lúdico como parte de la formación integral, posibilitando, así el adquirir libremente los conocimientos que le permitan al individuo la elección de oportunidades, para poder elegir aquellos factores que le produzcan el disfrute de un mejor nivel de vida, el cual consiste en la libertad que las personas gozan para elegir entre distintas opciones y formas de vida (2015, p. 10).

Ya que a través del juego los niños pueden proyectar sus deseos, miedos, emociones que no pueden expresar fácilmente con palabras y permite la evolución de las dimensiones del ser, aprendiendo a vivir y convivir con el otro, aceptando las diferencias, particularidades e individualidades para el respeto hacia el otro, como elemento fundamental de la educación del siglo XXI.

Tal como lo expresa Guerrero (2011), desarrollar la práctica de los valores para fomentar la sana convivencia, gestada en la familia y en el contexto social, minimizan los obstáculos que se le puedan presentar a los niños a lo largo de la vida. Esta práctica potencia, además, la formación de excelentes líderes. En este sentido, el fortalecimiento del liderazgo través del uso de herramientas lúdicas fortalecen las relaciones, inter, intra y sociogrupal.

Por lo tanto, se pretende articular la lúdica como estrategia dinamizadora para desarrollar las conductas prosociales del estudiante que le faciliten relacionarse de forma pacífica y armoniosa con sus compañeros, llevando buenas relaciones dentro y fuera del aula, de tal forma que a partir de actividades divertidas se reflejen actitudes y comportamientos en relación con la sana convivencia con su prójimo, lo que permitirá la aparición de lazos de afecto donde se expresarán los valores como el respeto, la tolerancia, la solidaridad, el amor hacia los demás, entre otros, fortaleciendo así la convivencia pacífica, desde el establecimiento de las reglas y la autonomía.

Entre algunas de las actividades lúdicas, se encuentra el juego, que para Vigostky (citado en Tripero, 2011), en su teoría constructivista sobre este concepto, considera que es la oportunidad que se le da al niño o niña de construir su aprendizaje y su propia realidad social y cultural, lo que le permite ampliar su capacidad de comprender la realidad de su entorno social natural. El juego como aprendizaje natural proporciona al estudiante motivación intrínseca para el desarrollo de sus capacidades de aprendizaje.

Por su parte, López (2008) considera que el juego es "una actividad propia de todos los animales evolucionados que posibilita y facilita su crecimiento como individuos singulares y sociales" (citado en Ruiz, 2017, p. 9). Por tanto, se puede considerar el juego como una actividad imprescindible para la evolución cognitiva, afectiva y social del niño y es necesario para un desarrollo integral. De esta manera, se puede considerar que el juego y la lúdica generan espacios agradables de aprendizaje y de moldeamiento de conductas, transforman el ambiente hostil en un espacio acogedor e interesante para los estudiantes, es decir que lo que allí se realiza no está dependiendo del cumplimiento de reglas, sino que abre la oportunidad de comunicación y participación en su propio aprendizaje.

La violencia entre pares, los conflictos en el aula, las burlas, los apodos, las agresiones, físicas, el rechazo, la exclusión, son algunos de los problemas que se presentan a diario, durante el proceso enseñanza-aprendizaje en las aulas, lo que afecta el rendimiento académico generando un bajo desempeño. En la medida en que se establezcan acciones que permitan la sana convivencia entre los educandos, se fortalecerán las relaciones sociales, la afirmación de roles, el liderazgo, la toma de decisiones, la autoestima, las habilidades de pensamiento y las habilidades motrices, los cuales son algunos aspectos que 
se desarrollan favorablemente a través del juego y la lúdica.

\section{METOdOLOGÍA}

La presente investigación se fundamenta dentro del paradigma sociocrítico, el cual responde a la intención de la ciencia social, que no es puramente empírica ni descriptiva, este paradigma, tiene por objetivo, según Montenegro, Román, Narváez y Gaviria (2006), el "análisis de las transformaciones sociales para dar respuesta a determinados problemas generados por estas" (p. 64). Se pretende que, a partir del reconocimiento de las conductas disruptivas reales, se pueda a través de la implementación de la estrategia transformar la realidad de las conductas y mejorar la praxis educativa, optimizando de esta manera el proceso enseñanza-aprendizaje dentro del aula.

Es una investigación de enfoque introspectivo-vivencial que, según Pulgar, Manzanero, Soto, Matos y Capote (2014), es una interpretación de las características socioculturales, donde se aborda la realidad humana y social, interpretando y comprendiendo la realidad como aparece en el contexto.

En este sentido, a partir de la interpretación de la realidad tal y como se presenta, se busca generar espacios de concientización en los estudiantes a partir de las experiencias lúdicas vividas, sentidas y compartidas con los demás, lo cual le permitirá adquirir nuevas conductas.

De la misma forma, es de tipo cualitativo, este tipo de investigación, según Hernández, Fernández y Baptista (2014), "proporciona profundidad a los datos, dispersión, riqueza interpretativa, contextualización del ambiente o entorno, detalles y experiencias únicas. También aporta un punto de vista 'fresco, natural y completo' de los fenómenos, así como flexibilidad" (p. 20). Tiene como propósito "reconstruir" la realidad tal y como la observan los actores de un sistema social previamente definido; así mismo, para confirmar si la teoría es apoyada por los hechos, el investigador comienza examinando el mundo social y en este proceso desarrolla una teoría "consistente" con la que observa qué ocurre, lo que permite la recopilación de información necesaria para conocer profundamente el problema social de esta comunidad desde su propio contexto.
De esta manera, se profundiza en la problemática que están presentando y realizan una serie de actividades lúdicas, donde la participación genere cambios positivos en los individuos, además de entender y ahondar en los fenómenos a partir de la perspectiva de los agentes que participan desde su medio natural; donde la investigación se dirige a la comprensión de experiencias en un medio específico y se hace necesaria la identificación, interpretación y análisis de la realidad natural de los comportamientos prosociales.

El abordaje de la presente investigación, de acuerdo con Elliot (1993), corresponde a un diseño de investigación-acción (IA), que se define como "un estudio de una situación social con el fin de mejorar la calidad de la acción dentro de la misma" (citado por Tatar y Vargas, 2017). A partir de un proceso de observación de la realidad, se busca generar acciones que transmuten las conductas disruptivas en prosociales, a partir de la implementación de la estrategia metodológica que pretende hacer más eficaz la práctica educativa, promoviendo estas conductas, las cuales, desde la reflexión, la crítica y la ejecución, buscan transformar la práctica educativa incidiendo en el cambio de actitudes de los educandos.

En la fase I se buscó caracterizar el nivel de apropiación de las conductas prosociales en la población a través de la técnica de la observación directa y participante.

La fase II permitió la aplicación de las actividades lúdicas para fortalecer las conductas prosociales y minimizar las conductas disruptivas de los estudiantes, esta fase se desarrolla a la luz del paradigma critico social, aquí se observan, analizan y estudian las conductas reales que se generan en el grupo y mediante la implementación de las actividades para transformar los comportamientos observables (tabla 1).

Entre las actividades que comprendieron la fase II, se destacan los juegos de roles; actividades espirituales, como devocionales, que permiten la reflexión de los diferentes versículos que hacen referencia a la importancia de ayudar y cooperar con los demás; mesas redondas en donde los estudiantes den a conocer la forma como actuarían frente a situaciones que involucren ayudar o cooperar con el prójimo; juegos lúdicos y deportivos que faciliten la actividad física y el trabajo cooperativo; competencias grupales e 
individuales y estímulos entre compañeros, entre otras, que apunten al fortalecimiento de la conductas prosociales. Para la recolección de la información de cada actividad individual o grupal, se utilizó una lista de chequeo y un diario de campo y se realizaron siete sesiones de actividades lúdicas.

En la fase III, se reflexionó sobre los cambios en las conductas prosociales de los estudiantes que se generaron a partir del desarrollo y la finalización de las actividades aplicadas.

\section{RESULTADOS}

A partir del desarrollo e implementación del presente proyecto de investigación, donde se busca fortalecer las conductas prosociales a través de la lúdica en estudiantes de cuarto grado de una institución de carácter privado y luego de la realización de actividades, se obtuvieron una serie de resultados desde el análisis e interpretación de la información recolectada para la consecución de hallazgos (tabla 1).

Tabla 1. Estrategias lúdicas y resultados

\begin{tabular}{|c|c|}
\hline Estrategias implementadas & Resultados \\
\hline Proceso de sensibilización & $\begin{array}{l}\text { Se pudo evidenciar que los estudiantes tienen algunos } \\
\text { preconceptos claros sobre lo que son las conductas de ayuda. }\end{array}$ \\
\hline Estrategia 1: Juego rompe hielo & $\begin{array}{l}\text { Se pudo palpar que los estudiantes están deseosos de } \\
\text { explorar, descubrir y cambiar el ambiente escolar. }\end{array}$ \\
\hline $\begin{array}{l}\text { Estrategia 2: ¿Qué es una } \\
\text { conducta prosocial? }\end{array}$ & $\begin{array}{l}\text { Se pudo evidenciar que la mayoría de los estudiantes actuarían de } \\
\text { manera correcta o en otros términos más específicos prosocialmente, } \\
\text { a través de situaciones de la vida cotidiana, sin embargo, algunos } \\
\text { manifestaron la posición contraria con actitudes disruptivas. }\end{array}$ \\
\hline Estrategia 3: Ojos vendados & $\begin{array}{l}\text { A través de la presentación de videos, cuyos mensajes dejaban } \\
\text { una enseñanza, se reafirmó el valor de la escucha. Los estudiantes } \\
\text { interiorizaron la importancia de escuchar y ser escuchados. }\end{array}$ \\
\hline $\begin{array}{l}\text { Estrategia 4: Lectura tituladas "Los } \\
\text { tres toros" y "Amigos de verdad" }\end{array}$ & $\begin{array}{l}\text { Se evidencia que los estudiantes reconocen cuáles serían } \\
\text { las acciones positivas que colocarían en práctica ante } \\
\text { cualquier situación que implique ayudar a un amigo. }\end{array}$ \\
\hline Estrategia 5: Paseo por el lago encantado & $\begin{array}{l}\text { Se pudo observar que los estudiantes necesitan mayor motivación, en } \\
\text { la parte socioafectiva y en el autoconcepto, teniendo en cuenta que } \\
\text { las relaciones intrapersonales se asocian con las interpersonales. }\end{array}$ \\
\hline Estrategia 6: ¿Quién quiere ser prosocial? & $\begin{array}{l}\text { Se evidencia que en la medida que se les han desarrollado y } \\
\text { trabajado las conductas prosociales por medio de las actividades, } \\
\text { estos han podido interiorizar el concepto e incluso identificar las } \\
\text { diferentes situaciones de la vida en las que estas se presentan. }\end{array}$ \\
\hline Estrategia 7: Desafío prosocial & $\begin{array}{l}\text { Se pudo observar un cambio favorable en el comportamiento de los } \\
\text { estudiantes, como por ejemplo, la mejora de las conductas dentro } \\
\text { y fuera del aula, permitiendo un clima escolar más agradable, lo } \\
\text { que incide favorablemente en su desempeño académico. }\end{array}$ \\
\hline
\end{tabular}

Fuente: Magdaniel y Silvera (2014).

Las actividades de reflexión a través de los videos, sobre conductas prosociales reafirman en los estudiantes acciones como "ayudar es hacer cosas buenas por los demás", "ayudar es algo que hacen las personas buenas", "ayudar es importante para poder vivir en paz", "si ayudamos a las demás personas, estamos haciendo nuestra vida más fácil", "todos en algún momento ayudamos y a veces ni nos damos cuenta”, "trabajar en equipo hace que terminemos más rápido las cosas”. 
Además, para retroalimentar la enseñanza de los videos y fomentar en los estudiantes el liderazgo como valor, se realizó una actividad llamada "Soy un buen líder". En esta, los diferentes grupos presentaron situaciones que evidenciaron la importancia del saber escuchar y si realmente son buenos líderes, ya que uno de los equipos no cumplió con el reto, lo que les permitió a ellos mismos sacar sus propias conclusiones acerca del comportamiento y las acciones que debieron haber puesto en práctica, muchos dijeron que no escuchaban lo que el otro compañero decía (el guía) y otros que la persona que los estaba guiando no lo sabía hacer.

Durante el desarrollo de los juegos rompe hielo, los estudiantes demostraron el interés por trabajar en equipo, además se observaron conductas prosociales de ayuda y cooperación entre un equipo y otro. Algunos grupos lograron encontrar canales comunicativos para colocarse de acuerdo, mientras que a otros se les hacía bastante difícil; sin embargo, siempre terminaban la actividad de manera satisfactoria.

En este marco de ideas, se puede analizar que los estudiantes perciben la necesidad de aprender a ser buenos líderes y escucharse los unos con los otros y así mismo valoran la importancia que traen estos aspectos para su vida. Este resultado está fundamentado en las investigaciones sobre la inteligencia emocional de Goleman, citado por Jiménez (2005), quien identificó:

El arte de saber escuchar entre las principales habilidades de las personas con altos niveles de inteligencia emocional. La escucha es considerada como la primera de las aptitudes que determinan el manejo de las relaciones, lo que posibilita comprender a los demás, en lo que se incluye percibir sentimientos y perspectivas ajenas, e interesarse activamente por sus preocupaciones (p. 6).

En la siguiente actividad, denominada "Paseo por el lago encantado", se observó que al iniciar esta, los estudiantes vuelven a manifestar su interés por trabajar en grupo, pero con compañeros en específico, lo que se hizo fue que se organizaron los equipos de manera aleatoria para estimular la convivencia.

Una vez organizados los grupos y socializadas las reglas del juego, se les preguntó a los estudiantes qué expectativas tenían frente a la actividad, estos respondieron: "No sabemos si podremos ganar porque hay unos más débiles que otros”, "¡no es justo aquel equipo tiene mejores integrantes!", "si nos ponemos de acuerdo, sí podemos ganar", "es divertido jugar en grupos", entre otros comentarios.

Un resultado importante en el primer momento se evidenció cuando los compañeros de los equipos en vez de juzgar al participante que estuviera atrasado le gritaban estrategias para que este pudiera seguir adelante, allí se vivencia la conducta prosocial no altruista y de cooperación. Además, en el segundo momento se pudo observar cómo entre equipos trabajan mejor y mucho más rápido, concentrándose en cooperar para alcanzar el objetivo y no en juzgarse los unos a los otros cuando se equivocan.

Después de observar y evaluar esta actividad, se puede establecer que durante las competencias los estudiantes deben aprender a aceptar que perdieron y aprender de ello. Se hizo necesario trabajar y fortalecer la temática del manejo de las emociones, para ello se realizó una actividad donde se utilizó como estrategia la observación de la película llamada Intensamente, en donde se hizo evidente que a pesar de que la mayoría de estudiantes manifestaron que ya la habían visto y que les gustó, observaron atentamente la película, respondieron favorablemente las preguntas y dieron a conocer su opinión sobre cómo las emociones los dominaban en algunos momentos de sus vidas y no lo sabían controlar.

Algunas de las respuestas fueron: "Todos tenemos estas emociones todos los días", "todas las emociones son necesarias", "a la mayoría de nosotros casi siempre nos domina la furia y el desagrado", "si nuestra emoción dominante fuera la alegría no peleáramos tanto", "la tristeza a veces es necesaria para poder aprender lecciones", "me gusta la furia porque es fuerte", entre otras.

\section{DISCUSIÓN}

Con base en los resultados obtenidos, a partir de la encuesta de percepción realizada a los estudiantes, se puede establecer la importancia de la implementación de la estrategia lúdica para el fortalecimiento de las conductas prosociales, ya que estas favorecen los procesos de enseñanza-aprendizaje que se desarrollan dentro y fuera del aula. En este sentido, se hace necesario reconocer que el hombre es un ser social y 
que necesita de sus semejantes para vivir, para compartir sus emociones y resolver sus necesidades en situaciones cotidianas.

De la misma forma, se puede analizar el reconocimiento de las emociones que los estudiantes viven en la cotidianidad, realizando un proceso de reflexión que es muestra de los resultados del proceso de entrenamiento de habilidades prosociales (propios del presente trabajo de investigación. Es importante indicar que se está mejorando el control de sus emociones, esto lleva a mencionar el concepto de inteligencia emocional de Goleman, 1995), quien la define como la forma de interactuar con el mundo que tiene muy en cuenta los sentimientos, y engloba habilidades tales como el control de los impulsos, la autoconciencia, la motivación, el entusiasmo, la perseverancia, la empatía, la agilidad mental.

Los estudiantes manifestaron que si se le da el manejo correcto a las emociones se puede sacar ventaja de cada una de las situaciones que ocurren en el diario vivir, por lo tanto, se considera la importancia de la inclusión de la lúdica como estrategia en los diversos momentos de formación académica, facilitando que el aprendizaje sea divertido y natural.

En este sentido, la acción educativa del maestro se fortalece a través de la implementación de procedimientos que busquen dinamizar los procesos en la medida que se logren cambios conductuales en el estudiante, a partir de su motivación intrínseca hacia nuevas experiencias de aprendizaje, que es la base fundamental para alcanzar los logros propuestos

\section{CONCLUSIONES}

Los aportes o conclusiones más significativas que dejaron cada grupo fueron las siguientes: "Podemos elegir también la tristeza, no todo en la vida es felicidad, por medio de la tristeza podemos aprender cosas para la vida", "en los días en que todo nos desagrada y esta emoción nos domina, debemos respetar los gustos de los demás, porque no estamos solos en el mundo y todos pensamos diferente". Esto quiere decir que los estudiantes aprendieron, primero, a identificar que existen diferentes emociones y que estas surgen a partir de las situaciones que se presentan cada día, segundo, a reconocerse como seres sociales que hacen parte de una comunidad en donde existen diversas opiniones y gustos que deben aprender a respetar a pesar de no compartirlas y, tercero, que la alegría es el sentimiento dominante al momento de ayudar a los demás, ya que esta produce una sensación de satisfacción y goce por el hecho de sentirse útiles para las otras personas.

Como actividad 6 se realizó un juego llamado “¿Quién quiere ser prosocial?", en donde los estudiantes se mostraron bastante a la expectativa y se evidenció cómo al momento de dárseles las instrucciones y pedirles que realizaran grupos, a estos se les hizo muy fácil, no hubo discusiones, solamente se organizaron y estaban deseosos de seguir con la actividad.

En del desarrollo del segundo momento de la actividad, ocurrió un suceso bien interesante y significativo para este estudio, ya que los estudiantes mostraron que lograron entender, interiorizar y crear su propio concepto de lo que son las conductas prosociales, cómo identificarlas y ponerlas en práctica, demostrándolo por medio de carteleras que ellos mismos realizaron, en las cuales dieron ejemplos por medio de imágenes, incluso algunos propios, y luego socializaron a sus compañeros.

Los niños mediante la actividad realizada pusieron en práctica un aprendizaje constructivista y una retroalimentación de los conceptos que se han enfatizado durante la implantación de las actividades lúdicas, poniendo en evidencia la teoría de Echeverri y Gómez (2009), quienes afirman que "la lúdica fomenta el desarrollo psicosocial, la adquisición de saberes y da herramientas para consolidar la personalidad, todo a través de una amplia gama de posibilidades que interactúan el gozo, el placer, la creatividad y el conocimiento" (p. 4). Las estrategias fundamentadas en el método lúdico fomentan un aprendizaje significativo en los estudiantes, a partir del desarrollo de su motivación intrínseca.

Para la última y séptima actividad, se hizo necesario crear un ambiente, en el cual los estudiantes pusieron en práctica todas las conductas prosociales desarrolladas y fortalecidas en el transcurso de la implementación del presente proyecto. Esto se hizo a través de un "desafío prosocial", durante su desarrollo se obtuvieron resultados realmente significativos que no solo fueron percibidos por las investigadoras, sino también por los niños y la psicóloga, quien se 
encontraba presente durante el desarrollo de dicha actividad.

En correspondencia al logro del objetivo general, se puede concluir que a través del fortalecimiento de las conductas prosociales, se genera en los estudiantes cambios conductuales que se ven reflejados tanto convivencial como académicamente.

\section{REFERENCIAS}

Auné, S., Blum, D., Abal, J., Lozzia, G., y Horacio, F. (2014). La conducta prosocial: estado actual de la investigación. Perspectivas en Sicología, 11(2), 21-33.

Casado, P. (1984). Guía para una vida plena. Barcelona: Círculo de Lectores.

Costa, L. (2013). La inteligencia emocional según Goleman. https://sobretodolavida.com/2013/07/03/ la-inteligencia-emocional-segun-goleman/

Domínguez, C. (2015). La lúdica: una estrategia pedagógica depreciada. Ciudad de Juárez: Universidad Autónoma de Ciudad de Juárez. http://www3.uacj.mx/DGDCDC/ SP/Documents/RTI/2015/ICSA/La\%20ludica.pdf

Echeverri, J., y Gómez, J. (2009). Lo lúdico como componente de lo pedagógico, la cultura, el juego y la dimensión humana. http://blog.utp.edu.co/areaderecreacionpcdyr/files/2012/07/LO-LUDICO-COMO-COMPONENTE-DE-LO-PEDAGOGICO.pdf

Espinoza, L. y Rodríguez. R. (2017). La generación de ambientes de aprendizaje: un análisis de la percepción juvenil. Ride. Revista Iberoamericana para la Investigación y el Desarrollo Educativo, 7(14), 110-132. https:// dx.doi.org/10.23913/ride.v7i14.276

Goleman, D. (1995). La inteligencia emocional. LeLibros. http://ciec.edu.co/wp-content/uploads/2017/08/ La-Inteligencia-Emocional-Daniel-Goleman-1.pdf

Guerrero, H. (2011). El impacto del líder comunitario en el siglo XXI. Barranquilla: Editorial cuc.

Hernández, R., Fernández, C., y Baptista, P. (2006). Metodología de la investigación. https://periodicooficial. jalisco.gob.mx/sites/periodicooficial.jalisco.gob.mx/ files/metodologia_de_la_investigacion_-_roberto_hernandez_sampieri.pdf
Jiménez, C. (2005). La inteligencia lúdica. Juego y neuropedagogía en tiempos de transformación. Bogotá: Cooperativa Editorial del Magisterio.

López, I. (2018). La actividad científica en 4 de Eso, a través de métodos de enseñanza basados en el juego: juegos educativos y gamificación. http://digibug.ugr.es/ handle/10481/52122

Moñivas, A. (1996). La conducta prosocial. Cuadernos de trabajo social, 9, 125-142. revistas.ucm.es/index.php/ CUTS/article/viewFile/CUTS9696110125A/8357

Magdaniel, M., y Silvera, B. (2014). La metodología lúdica como dinamizador de las conductas prosociales. [Tesis de grado, Universidad de la costa]. Repositorio institucional cuc. http://hdl.handle.net/11323/394

Pulgar, R., Manzanero, R., Soto, A., Matos, A. y Suárez, E. (2014). Tendencias Epistemológicas de las investigaciones en los programas académicos de la División de estudios para graduados de la facultad de ciencias Económicas y Sociales De la Universidad de Zulia. Orbis. Revista Científica Ciencias Humanas, 10(29), 6-36. http://www.redalyc.org/comocitar. oa?id=70932556002

Ruiz, M. (2017). El juego: una herramienta importante para el desarrollo integral del niño en Educación Infantil. [Tesis de grado, Universidad de Cantabria]. Repositorio institucional Unican https://repositorio.unican. es/xmlui/bitstream/handle/10902/11780/RuizGutierrezMarta.pdf?sequence $=1$

Tatar, F., y Vargas, J. (2017). La investigación acción participante: una oportunidad para la transformación de la cátedra de paz en la universidad. Revista Ciudad Paz-ando, 10(2), 40-53. https://doi. org/10.14483/2422278X.12716

Tripero, A. (2011). Vigostsky y su teoría constructivista del juego. http://webs.ucm.es/BUCM/revcul/e-learning-innova/5/art382.php\#.XUdGb45KjIV 